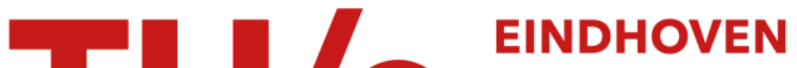 UNIVERSITY OF TECHNOLOGY
}

\section{Multigrid applied to mixed finite element schemes for current continuity equations}

\section{Citation for published version (APA):}

Reusken, A. A. (1990). Multigrid applied to mixed finite element schemes for current continuity equations. (RANA : reports on applied and numerical analysis; Vol. 9013). Technische Universiteit Eindhoven.

Document status and date:

Published: 01/01/1990

\section{Document Version:}

Publisher's PDF, also known as Version of Record (includes final page, issue and volume numbers)

\section{Please check the document version of this publication:}

- A submitted manuscript is the version of the article upon submission and before peer-review. There can be important differences between the submitted version and the official published version of record. People interested in the research are advised to contact the author for the final version of the publication, or visit the $\mathrm{DOI}$ to the publisher's website.

- The final author version and the galley proof are versions of the publication after peer review.

- The final published version features the final layout of the paper including the volume, issue and page numbers.

Link to publication

\section{General rights}

Copyright and moral rights for the publications made accessible in the public portal are retained by the authors and/or other copyright owners and it is a condition of accessing publications that users recognise and abide by the legal requirements associated with these rights.

- Users may download and print one copy of any publication from the public portal for the purpose of private study or research.

- You may not further distribute the material or use it for any profit-making activity or commercial gain

- You may freely distribute the URL identifying the publication in the public portal.

If the publication is distributed under the terms of Article 25fa of the Dutch Copyright Act, indicated by the "Taverne" license above, please follow below link for the End User Agreement:

www.tue.nl/taverne

Take down policy

If you believe that this document breaches copyright please contact us at:

openaccess@tue.nl

providing details and we will investigate your claim. 
RANA 90-13

November 1990

MULTIGRID APPLIED TO MIXED

FINITE ELEMENT SCHEMES FOR

CURRENT CONTINUITY EQUATIONS

by

A. Reusken

Reports on Applied and Numerical Analysis

Department of Mathematics and Computing Science

Eindhoven University of Technology

P.O. Box 513

$5600 \mathrm{MB}$ Eindhoven

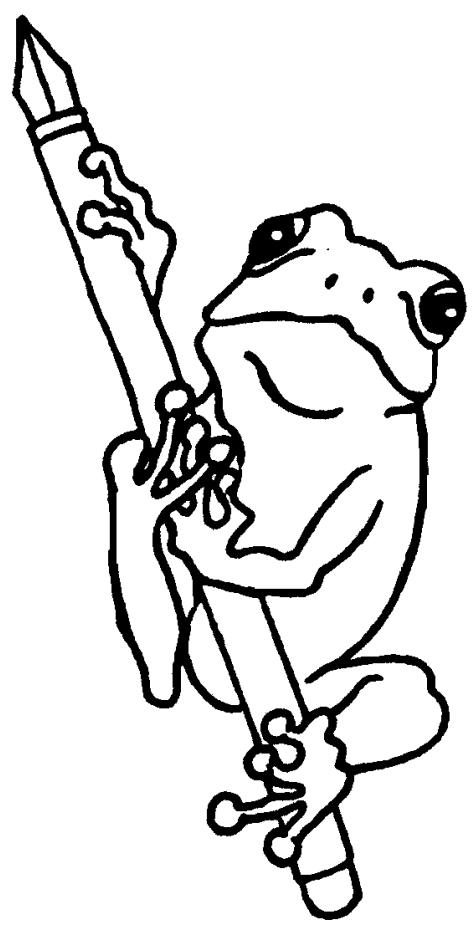

The Netherlands 


\title{
MULTIGRID APPLIED TO \\ MIXED FINITE ELEMENT SCHEMES \\ FOR CURRENT CONTINUITY EQUATIONS
}

by

\author{
Arnold Reusken
}

\begin{abstract}
Mixed finite element discretizations for current continuity equations are presented in $[5,6,8]$. We consider the resulting system of equations and develop a multigrid method for such a system. Our method is based on a connection between the given system and nonconforming Crouzeix-Raviart finite elements.
\end{abstract}

\section{Introduction}

Recently (new) mixed finite element discretizations for current continuity equations have been presented in $[5,6,8]$. These schemes have nice properties: They provide an $M$-matrix, there is current conservation, and a good approximation of sharp shapes. Such a scheme results in a large sparse system of equations for the unknowns. A "standard" multigrid solver cannot be used for this system due to the presence of (extremely) large convection in part of the domain and the use of mixed finite elements. In this paper we present a suitable multigrid method for solving this system. The method is based on a connection between the system resulting from the mixed FE discretization and nonconforming Crouzeix-Raviart FE. Using this connection together with multigrid theory for nonconforming FE then leads to a multigrid method for the given system. These ideas underlying our method are an important subject of this paper and might be useful in other situations too.

We consider the continuous problem as in [6]:

$$
\left\{\begin{array}{cl}
\text { find } u \in H^{1}(\Omega) & \text { such that } \\
\operatorname{div}(\nabla u+u \nabla \psi)=f & \text { in } \Omega \subset \mathbb{R}^{2} \\
u=g & \text { on } \Gamma_{0} \subset \partial \Omega \\
\frac{\partial u}{\partial n}+u \frac{\partial \psi}{\partial n}=0 & \text { on } \Gamma_{1}=\partial \Omega \backslash \Gamma_{0}
\end{array}\right.
$$


In this current continuity equation (the current is defined by $\mathbf{J}=\nabla u+u \nabla \psi$ ) we assume that $\psi$ is a given function.

In $\$ 2$ we collect some results from [6] concerning the mixed FE discretization of (1.1). In $\$ 3$ we discuss a connection between the system resulting from $\$ 2$ and a suitable variational problem in the Crouzeix-Raviart nonconforming FE space. In $\$ 4$ we present our multigrid method. In $\$ 5$ we give some numerical results.

\section{Mixed finite element schemes for current continuity equations}

In this section we collect some results from $[5,6]$.

2.1. Continuous problem. The problem we want to solve is given in (1.1). Using the Slotboom variable $\rho:=e^{\psi} u$ this results in the following problem

$$
\left\{\begin{array}{cc}
\text { find } \rho \in H^{1}(\Omega) & \text { such that } \\
\operatorname{div}\left(e^{-\psi} \nabla \rho\right)=f & \text { in } \Omega \\
\rho=\chi:=e^{\psi} g & \text { on } \Gamma_{0} \\
\frac{\partial \rho}{\partial n}=0 & \text { on } \Gamma_{1}
\end{array}\right.
$$

2.2. Discretization. For ease we assume that $\Omega$ is a polygonal domain. Let $\left\{T_{k}\right\}_{k \geq 0}$ be a regular sequence of triangulations of $\Omega$ into triangles $T$. The set of edges of $T_{k}$ is denoted by $E_{k}$; edges are denoted by $e_{i}$ and $E_{k}=\left\{e_{i}\right\}_{i \in I_{0} \cup I}$ with $I_{0}$ the index set of edges $e_{i} \subset \Gamma_{0}$ and $I$ the index set of edges $e_{i} \subset \bar{\Omega} \backslash \Gamma_{0}$. Midpoints of edges are denoted by $m_{i}$.

We use the lowest order Raviart-Thomas mixed finite element method to discretize (2.2). Set

$$
R T(T)=\left\{\tau=\left(\tau_{1}, \tau_{2}\right) \mid \tau_{1}=\alpha+\beta x, \tau_{2}=\gamma+\beta y, \alpha, \beta, \gamma \in \mathbb{R}\right\} \quad\left(T \in T_{k}\right)
$$

and define

$$
\begin{aligned}
& V_{k}=\left\{\tau \in\left(L^{2}(\Omega)\right)^{2} \mid \operatorname{div} \tau \in L^{2}(\Omega), \boldsymbol{\tau} \cdot \mathbf{n}=0 \text { on } \Gamma_{1}, \tau_{\mid T} \in R T(T) \text { for all } T \in T_{k}\right\}, \\
& W_{k}=\left\{\varphi \in L^{2}(\Omega) \mid \varphi_{\mid T} \in P_{0}(T) \text { for all } T \in T_{k}\right\} .
\end{aligned}
$$

Then the discretization of (2.2) is as follows

$$
\left\{\begin{array}{l}
\text { find } \mathbf{J}_{k} \in V_{k} \text { and } \rho_{k} \in W_{k} \text { such that } \\
\int_{\Omega} e^{\psi} \mathbf{J}_{k} \cdot \tau d \mathbf{x}+\int_{n} \rho_{k} \operatorname{div} \tau d \mathbf{x}=\int_{\Gamma_{0}} \chi \tau \cdot \mathbf{n} d \Gamma \quad \forall \tau \in V_{k} \\
\int_{\Omega}^{n} \varphi \operatorname{div} \mathbf{J}_{k} d \mathbf{x}=\int_{n} f \varphi d \mathbf{x} \quad \forall \varphi \in W_{k} .
\end{array}\right.
$$


The matrix associated with (2.3) is not positive definite. To circumvent this Lagrange multipliers are used.

Define $\tilde{V}_{k}=\left\{\tau \in\left(L^{2}(\Omega)\right)^{2} \mid \tau_{\mid T} \in R T(T)\right.$ for all $\left.T \in T_{k}\right\}$, and for $\zeta \in L^{2}\left(\Gamma_{0}\right)$ $\Lambda_{k, 6}=\left\{\mu \in L^{2}\left(E_{k}\right) \mid \mu_{k e} \in P_{0}(e)\right.$ for all $e \in E_{k}, \int_{e_{i}}(\mu-\zeta) d s=0$ for all $\left.i \in I_{0}\right\}$. Instead of (2.3) we now consider the discretization

$$
\left\{\begin{array}{l}
\text { find } \tilde{\mathbf{J}}_{k} \in \tilde{V}_{k}, \quad \tilde{\rho}_{k} \in W_{k}, \tilde{\lambda}_{k} \in \Lambda_{k, x} \text { such that } \\
\int_{\boldsymbol{\Omega}} e^{\psi} \tilde{\mathbf{J}}_{k} \cdot \tau d \mathbf{x}+\sum_{T} \int_{T} \tilde{\rho}_{k} \operatorname{div} \tau d \mathbf{x}-\sum_{T} \int_{\partial T} \tilde{\lambda}_{k} \tau \cdot \mathbf{n} d s=0 \quad \forall \tau \in \tilde{V}_{k} \\
\sum_{T} \int_{T} \varphi \operatorname{div} \tilde{\mathbf{J}}_{k} d \mathbf{x}=\int_{\Omega} f \varphi d \mathbf{x} \quad \forall \varphi \in W_{k} \\
\sum_{T} \int_{\partial T} \mu \tilde{\mathbf{J}}_{k} \cdot \mathbf{n} d s=0 \quad \forall \mu \in \Lambda_{k, 0} .
\end{array}\right.
$$

The problem (2.4) has a unique solution and $\tilde{\mathfrak{J}}_{k} \equiv \mathbf{J}_{k}, \tilde{\rho}_{k} \equiv \rho_{k}$ holds. Moreover $\tilde{\lambda}_{k}$ is a good approximation of $\rho$ at the interelements (see [1]). In the resulting matrix-vector problem the unknowns corresponding to $\tilde{\mathbf{J}}_{k}$ and $\tilde{\rho}_{k}$ can be eliminated by static condensation. Lemma 2.5 below shows that in (2.4) $\tilde{\mathbf{J}}_{k}$ and $\tilde{\rho}_{k}$ can be eliminated a-priori. The proof of this lemma is straightforward using the arguments concerning static condensation in [6].

Notation. In the remainder we use the following notation: for $h \in L^{2}(A)$ we set $\bar{h}_{\mid A}:=$ $\frac{1}{|A|} \int_{A} h(x) d x$.

Lemma 2.5. Define $\mathbf{J}^{f}$ by $\mathbf{J}_{\mid T}^{f}(\mathbf{x})=\frac{1}{2} \bar{f}_{\mid T} \mathbf{x}$. Define a symmetric bilinear form $b_{k}: L^{2}\left(E_{k}\right) \times$ $L^{2}\left(E_{k}\right) \rightarrow \mathbb{R}$ and a linear functional $F_{k}: L^{2}\left(E_{k}\right) \rightarrow \mathbb{R}$ as follows:

$$
\begin{aligned}
& b_{k}(\lambda, \mu)=\sum_{T}\left(|T| \overline{e^{\psi}} \mid T\right)^{-1} \int_{\partial T} \lambda \mathbf{n} d s \cdot \int_{\partial T} \mu \mathbf{n} d s \\
& F_{k}(\mu)=\sum_{T}\left(|T| \overline{e^{\psi}} \mid T\right)^{-1} \int_{T} e^{\psi} \mathbf{J}^{f} d \mathbf{x} \cdot \int_{\partial T} \mu \mathbf{n} d s-\sum_{T} \int_{\partial T} \mu \mathbf{J}^{f} \cdot \mathbf{n} d s .
\end{aligned}
$$

Then the solution $\tilde{\lambda}_{k}$ of (2.4) is also the unique solution of the following problem

$$
\left\{\begin{array}{l}
\text { find } \tilde{\lambda}_{k} \in \Lambda_{k, x} \text { such that } \\
b_{k}\left(\tilde{\lambda}_{k}, \mu\right)=F_{k}(\mu) \text { for all } \mu \in \Lambda_{k, 0} .
\end{array}\right.
$$

2.3. Rescaling. The Lagrange multiplier $\tilde{\lambda}_{k}$ is an approximation of $\rho=e^{\psi} u$, and is not suited for actual computation if the range of $\psi$ is large (which often happens in semiconductor 
problems); moreover we are interested in approximating $u$ instead of $\rho$. So we rescale $\tilde{\lambda}_{k}$ to get an approximation $\tilde{\mu}_{k}$ of $u$ (at the interelements).

In addition to $\Lambda_{k, 6}$ we will also use the space $\Lambda_{k}:=\left\{\mu \in L^{2}\left(E_{k}\right) \mid \mu_{\mid e} \in P_{0}(e)\right.$ for all $\left.e \in E_{k}\right\}$ with standard basis functions ( 1 on one edge and 0 on all other edges) denoted by $\left\{\mu_{i}\right\}_{i \in I_{0} \cup I}$.

We define the isomorphism $Q_{k}: \Lambda_{k, g} \rightarrow \Lambda_{k, \chi}\left(g, \chi\right.$ as in (2.2)) as follows. Take $\mu \in \Lambda_{k, g}$ then:

$$
\begin{array}{ll}
\text { for } e \subset \Gamma_{0} & \left(Q_{k} \mu\right)_{\mid e}=\bar{\chi}_{\mid e} \\
\text { for } e \subset \bar{\Omega} \backslash \Gamma_{0} & \left(Q_{k} \mu\right)_{\mid e}=\bar{e}_{\mid e}^{\bar{\psi}_{\mid e}} \mu_{\mid e} .
\end{array}
$$

Using this isomorphism we can rewrite (2.6):

$$
\left\{\begin{array}{l}
\text { find } \tilde{\mu}_{k} \in \Lambda_{k, \varrho} \text { such that } \\
b_{k}\left(Q_{k} \tilde{\mu}_{k}, \mu\right)=F_{k}(\mu) \text { for all } \mu \in \Lambda_{k, 0} .
\end{array}\right.
$$

The problem (2.7) is the final one, which we actually want to solve.

Rewriting (2.7) as a matrix-vector problem using the basis $\left\{\mu_{i}\right\}$ of $\Lambda_{k}$ yields the following system of equations for the unknowns $\left\{\alpha_{j}\right\}_{j \in I}$ with $\tilde{\mu}_{k}=\sum_{j \in I} \alpha_{j} \mu_{j}+\sum_{j \in I_{0}} \bar{g}_{\mid e_{j}} \mu_{j}$

$$
\sum_{j \in I} b_{k}\left(Q_{k} \mu_{j}, \mu_{i}\right) \alpha_{j}=-\sum_{j \in I_{0}} b_{k}\left(Q_{k} \mu_{j}, \mu_{i}\right) \bar{g}_{\mid e_{j}}+F_{k}\left(\mu_{i}\right) \quad \text { for } i \in I
$$

Remark 2.9. Expressions for $b_{k}\left(Q_{k} \mu_{j}, \mu_{i}\right)$ and $F_{k}\left(\mu_{i}\right)$ can be found in [6]. The resulting (nonsymmetric) matrix is an $M$-matrix if the triangulation is weakly acute (no angle $>\frac{\pi}{2}$ ). The above discretization has upwinding features for strong convection. For a discussion of this upwinding effect to refer to [6].

\section{Connection with nonconforming finite elements}

In this section we show that the system (2.8) corresponds to a variational problem in the (nonconforming) $P 1$ Crouzeix-Raviart finite element space.

Consider the Crouzeix-Raviart $(P 1)$ space corresponding to $T_{k}$ :

$S_{k}=\left\{v \in L^{2}(\Omega) \mid v_{\mid T}\right.$ is linear for all $T \in T_{k}, v$ is continuous at midpoints of edges $\}$. The standard basis of $S_{k}$ is denoted by $\left\{\varphi_{i}\right\}_{i \in I \cup I_{0}}\left(I, I_{0}\right.$ as in $\left.\S 2\right)$. For $\zeta \in L^{2}\left(\Gamma_{0}\right)$ we define $S_{k, \zeta}:=\left\{v \in S_{k} \mid v\left(m_{i}\right)=\bar{\zeta}_{\mid e_{i}} \forall i \in I_{0}\right\}$

We also use the space $\tilde{S}_{k}:=\left\{v \in L^{2}(\Omega) \mid v_{\mid T}\right.$ is linear for all $\left.T \in T_{k}\right\}$.

Let $R_{k}: S_{k} \rightarrow \tilde{S}_{k}$ be the linear operator which satisfies

$$
\left(R_{k} \varphi_{i}\right)_{\mid T}=\left(\bar{e}_{\mid T}\right)^{-1} \bar{e}_{\mid e_{i}} \varphi_{i} \text { for all basis functions } \varphi_{i} \text { and all } T \in T_{k} \text {. }
$$


Note that $\left(R_{k} v\right)_{\mid T}=v_{\mid T}$ if $\psi$ is constant on $T$.

Theorem 3.2. Let $f$ be as in (1.1) and define the function $G_{k}(f) \in L^{2}(\Omega)$ by $G_{k}(f)_{\mid T}=\bar{f}_{\mid T}\left(1 \frac{1}{2}-\frac{1}{2}\left(\bar{e}^{\phi} \mid T\right)^{-1} e^{\psi}\right)$. Consider the problem

$$
\left\{\begin{array}{l}
\text { find } \tilde{\eta}_{k} \in S_{k, g} \text { such that } \\
\sum_{T} \int_{T} \nabla\left(R_{k} \tilde{\eta}_{k}\right) \cdot \nabla \varphi d \mathrm{x}=-\int_{\Omega} G_{k}(f) \varphi d \mathrm{x} \text { for all } \varphi \in S_{k, 0}
\end{array}\right.
$$

Write $\tilde{\eta}_{k}=\sum_{j \in I} \alpha_{j} \varphi_{j}+\sum_{j \in I_{0}} \bar{g}_{\mid e_{j}} \varphi_{j}$. Taking $\varphi=\varphi_{i}(i \in I)$ in (3.3) results in a system of equations for the unknowns $\left\{\alpha_{j}\right\}_{j \in I}$ that is given in (2.8).

Proof. Use the notation $a_{k}(\eta, \varphi)=\sum_{T} \int_{T} \nabla\left(R_{k} \eta\right) \cdot \nabla \varphi d x$. Taking $\varphi=\varphi_{i}$ in (3.3) yields

$$
\sum_{j \in I} a_{k}\left(\varphi_{j}, \varphi_{i}\right) \alpha_{j}=-\sum_{j \in I_{0}} a_{k}\left(\varphi_{j}, \varphi_{i}\right) \bar{g}_{\mid e_{j}}-\int_{n} G_{k}(f) \varphi_{i} d \mathbf{x} \quad(i \in I)
$$

By comparing (3.4) with (2.8) it follows that we only have to show:

(a) $b_{k}\left(Q_{k} \mu_{j}, \mu_{i}\right)=a_{k}\left(\varphi_{j}, \varphi_{i}\right)$

(b) $\quad F_{k}\left(\mu_{i}\right)=-\int_{n} G_{k}(f) \varphi_{i} d \mathbf{x}$.

Using the definitions and checking per triangle it is clear that it is sufficient to prove:

$$
\begin{aligned}
& |T|^{-1} \int_{\partial T} \mu_{j} \mathrm{n} d s \cdot \int_{\partial T} \mu_{i} \mathrm{n} d s=\int_{T} \nabla \varphi_{j} \cdot \nabla \varphi_{i} d \mathbf{x} \\
& |T|\left(\overline{e^{\psi}} \mid T\right)^{-1} \int_{T} e^{\psi} \mathrm{J}^{f} d \mathbf{x} \cdot \int_{\partial T} \mu_{i} \mathrm{n} d s-\int_{\partial T} \mu_{i} \mathrm{~J}^{f} \cdot \mathrm{n} d s=-\int_{T} G_{k}(f) \varphi_{i} d \mathbf{x} .
\end{aligned}
$$

Let $T$ be a given triangle with edges $e_{1}, e_{2}, e_{3}$, midpoints of edges $m_{1}, m_{2}, m_{3}$ (with corresponding coordinate vectors $\left.\mathbf{M}_{1}, \mathbf{M}_{2}, \mathbf{M}_{3}\right)$ and unit outward normals $\mathbf{n}^{(1)}, \mathbf{n}^{(2)}, \mathbf{n}^{(3)}$. Define $\nu^{(i)}=\left|e_{i}\right| \mathbf{n}^{(i)}$. Now consider a basis function $\varphi_{i}$ which is 1 in $m_{i} \quad(i \in\{1,2,3\})$ and 0 in all $m_{j}$ with $j \neq i$. One easily verifies that $\varphi_{i \mid T}$ can be represented as

$$
\varphi_{i \mid T}(\mathbf{x})=|T|^{-1} \nu^{(i)} \cdot\left(\mathbf{x}-\mathbf{M}_{j}\right) \text { for } j \in\{1,2,3\} \backslash\{i\} .
$$

Below we also use the following well-known result 


$$
\text { for } p \in P_{2}(T) \text { we have } \int_{T} p(\mathbf{x}) d \mathbf{x}=\frac{1}{3}|T|\left(p\left(m_{1}\right)+p\left(m_{2}\right)+p\left(m_{3}\right)\right) \text {. }
$$

The lefthand side of (a') equals $|T|^{-1} \nu^{(j)} \cdot \nu^{(i)}$. Using (3.5) it follows that $\int_{T} \nabla \varphi_{j} \cdot \nabla \varphi_{i} d x=$ $|T|\left(|T|^{-1} \nu^{(j)}\right) \cdot\left(|T|^{-1} \nu^{(i)}\right)=|T|^{-1} \nu^{(j)} \cdot \nu^{(i)}$. So (a') holds.

The proof of (b') runs as follows

$$
\begin{aligned}
& \left(|T| \overline{e^{\psi}} \mid T\right)^{-1} \int_{T} e^{\psi} \mathrm{J}^{f} d \mathbf{x} \cdot \int_{\partial T} \mu_{i} \mathbf{n} d s-\int_{\theta T} \mu_{i} \mathbf{J}^{f} \cdot \mathbf{n} d s \\
& =\frac{1}{2} \bar{f}_{\mid T}\left\{|T|^{-1}\left(\bar{e}^{\phi} \mid T\right)^{-1} \int_{T} e^{\psi} \mathbf{x} \cdot \nu^{(i)} d \mathbf{x}-\frac{1}{\left|e_{i}\right|} \int_{e_{i}} \mathbf{x} \cdot \nu^{(i)} d s\right\} \\
& =\frac{1}{2} \bar{f}_{\mid T}\left\{|T|^{-1}\left(\overline{e^{\phi}} \mid T\right)^{-1} \int_{T} e^{\psi}\left(|T| \varphi_{i}+\nu^{(i)} \cdot \mathbf{M}_{j}\right) d \mathbf{x}-\frac{1}{\left|e_{i}\right|} \int_{e_{i}}|T| \varphi_{i}+\nu^{(i)} \cdot \mathbf{M}_{j} d s\right\} \quad \text { (use (3.5)) } \\
& =\frac{1}{2} \bar{f}_{\mid T}\left\{\int_{T}\left(\bar{e}^{\psi} \mid T\right)^{-1} e^{\psi} \varphi_{i} d \mathbf{x}+\nu^{(i)} \cdot \mathbf{M}_{j}-|T|-\nu^{(i)} \cdot \mathbf{M}_{j}\right\} \quad \text { (use } \varphi_{i} \equiv 1 \text { on } e_{i} \text { ) } \\
& =\frac{1}{2} \bar{f}_{\mid T}\left\{\int_{T}\left(\bar{e}^{\psi} \mid T\right)^{-1} e^{\psi} \varphi_{i} d \mathbf{x}-3 \int_{T} \varphi_{i} d \mathbf{x}\right\} \quad \text { (use (3.6)) } \\
& =-\int_{T} \bar{f}_{\mid T}\left(1 \frac{1}{2}-\frac{1}{2}\left(\bar{e}^{\bar{\psi}} \mid T\right)^{-1} e^{\psi}\right) \varphi_{i} d \mathbf{x} \\
& =-\int_{T} G_{k}(f) \varphi_{i} d \mathbf{x}
\end{aligned}
$$

Remark 3.7. The system (2.8) corresponds to the variational problem (2.7), for the rescaled Lagrange multipliers $\tilde{\mu}_{k}$, which results in a natural way by using the Slotboom variable and mixed finite elements. From Theorem 3.2 we conclude that the system (2.8) also corresponds to the rather special nonconforming finite element discretization in (3.3). The discretization in (3.3) can be related to the given continuous problem in (1.1) as follows. Consider (1.1) as a variational problem in $H^{1}$ with a bilinear form $a_{\Omega}(u, v)=\int_{n}(\nabla u+u \nabla \psi) \cdot \nabla v d \mathbf{x}=$ $\int_{\Omega} e^{-\psi} \nabla\left(e^{\psi} u\right) \cdot \nabla v d \mathbf{x}$ and a righthand side functional $v \rightarrow-\int_{\Omega} f v d \mathbf{x}$. The problem in (3.3) results from this by a modified discretization in the Crouzeix-Raviart $P_{1}$ space. The modification concerns the following:

(a) in the righthand side functional, $f$ is replaced by $\tilde{f}$ with $\tilde{f}_{\mid T}=\bar{f}_{\mid T}\left(1 \frac{1}{2}-\frac{1}{2}\left(\bar{e}^{\bar{\psi}} \mid T\right)^{-1} e^{\psi}\right)$ 
(b) $a_{\Omega}(u, v)$ is replaced by $\tilde{a}_{\Omega}(u, v)$ with $\tilde{a}_{T}\left(\varphi_{i}, \varphi_{j}\right)=\int_{T}\left(\overline{e^{\bar{\psi}}} \mid T\right)^{-1} \nabla\left(\left.\overline{e^{\psi}}\right|_{e_{i}} \varphi_{i}\right) \cdot \nabla \varphi_{j} d \mathbf{x}$ (so $e^{-\psi} \mid T$ is replaced by the harmonic average $\left(\bar{e}^{\phi} \mid T\right)^{-1}$ and $e^{\psi} u=\Sigma \alpha_{i} e^{\psi} \varphi_{i}$ is replaced by $\left.\Sigma \alpha_{i} \bar{e}^{\bar{\phi}} \mid e_{i} \varphi_{i}\right)$.

Other relations between mixed and nonconforming finite elements are discussed in [1].

\section{Multigrid method}

In this section we develop a multigrid method that can be used to solve the system (2.8). The method is based on the equivalence between (2.8) and (3.3). Through this equivalence we are led to multigrid methods for nonconforming finite elements (as in [2], [3,4]). Before we specify the multigrid method we first discuss

- triangulation

- sequence of bilinear forms

- prolongation

- smoother.

4.1. Triangulation. In the remainder we assume a regular sequence of triangulations $\left\{T_{k}\right\}_{k \geq 0}$ in which $T_{k}$ ("finer triangulation") is obtained from $T_{k-1}$ ("coarser triangulation") by connecting the midpoints of the edges of the triangles of $T_{k-1}$. We also assume that $T_{0}$ is weakly acute (all triangles have angles $\leq \frac{\pi}{2}$ ). In the numerical experiments in $\S 5$ we will use $\Omega=[0,1] \times[0,1]$ and a triangulation as in Figure 1 .

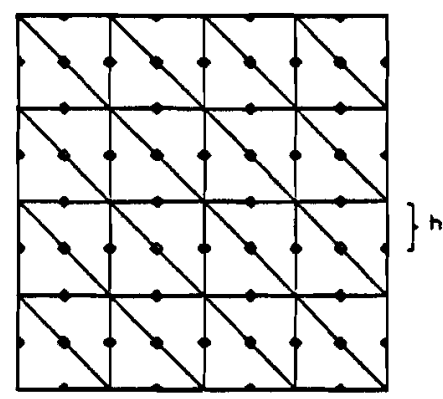

Figure 1.

4.2. Sequence of bilinear forms. We assume a given $k=k_{\max }$ for which we want to solve (2.8). We treat this problem in the (equivalent) form (3.3). For ease we use the notation $a_{k}(\eta, \varphi)=\sum_{T} \int_{T} \nabla\left(R_{k} \eta\right) \cdot \nabla \varphi d \mathbf{x} \quad\left(\eta, \varphi \in S_{k, 0}\right)$ and $\tilde{\eta}_{k}^{d}:=\sum_{j \in I_{0}} \bar{g}_{\left.\right|_{j}} \varphi_{j} ;$ then we can rewrite (3.3) as follows:

$$
\left\{\begin{array}{l}
\text { find } \eta_{k}^{*} \in S_{k, 0} \text { such that } \\
a_{k}\left(\eta_{k}^{*}, \varphi\right)=-a_{k}\left(\tilde{\eta}_{k}^{d}, \varphi\right)-\int_{\Omega} G_{k}(f) \varphi d \mathrm{x} \text { for all } \varphi \in S_{k, 0} .
\end{array}\right.
$$


If we take $k=k_{\max }$ then (4.1) results in the problem we want to solve. We make an obvious choice for approximation on coarser grids: We take the discrete operators induced by the bilinear form $a_{k}(\cdot, \cdot) \quad\left(0 \leq k<k_{\max }\right)$. We note, however, that it is not clear if a suitable "approximation property" (cf. [7]) holds for these coarse grid operators.

4.3. Prolongation. In multigrid methods we need mappings between the finite element spaces. In conforming finite elements the spaces are nested, so there is a natural imbedding of the coarse grid functions in the fine grid function space. In nonconforming finite elements this is not the case, so we need a suitable prolongation $P_{k}: S_{k-1,0} \rightarrow S_{k, 0}$. Such prolongations are given in [2], [3,4]. We use the prolongation as proposed in [2]: For $P_{k}$ we take the orthogonal projection w.r.t. the $L^{2}$-inner product of $S_{k-1,0}$ on $S_{k, 0}$. More information concerning this prolongation is given in Lemma 4.2 below. We omit the proof because it is straightforward.

Lemma 4.2. Define $W_{k}:=S_{k, 0} \oplus S_{k-1,0}$. Let $P_{k}$ be the orthogonal projection w.r.t. the $L^{2}$-inner product of $W_{k}$ on $S_{k, 0}$. Take $u \in W_{k}$ and take the midpoint $m_{i}$ of an edge $e_{i} \in E_{k}$. If $e_{i} \subset \partial \Omega$ then $\left(P_{k} u\right)\left(m_{i}\right)=u\left(m_{i}\right)$.

If $e_{i} \not \subset \partial \Omega$ then $\left(P_{k} u\right)\left(m_{i}\right)=\left(\left|T^{L}\right|+\left|T^{R}\right|\right)^{-1}\left(\left|T^{L}\right| u_{\mid T^{2}}\left(m_{i}\right)+\left|T^{R}\right| u_{\mid T^{R}}\left(m_{i}\right)\right)$, where $T^{L}, T^{R} \in T_{k}$ are the two triangles with edge $e_{i}$.

From Lemma 4.2 it is clear that if $u$ is continuous at $m_{i}$ then $\left(P_{k} u\right)\left(m_{i}\right)=u\left(m_{i}\right)$. For this $P_{k}$ formulas suited for computation can be found in [2].

4.4. Smoother. Systematic research concerning a suitable smoother for a system as in (2.8) has not been done yet. Two obvious candidates are ILU (which is a popular method for problems with strong convection) and Gauss-Seidel. Here we restrict ourselves to (a variant of) Gauss-Seidel. Numerical experiments for problems with a triangulation as in Fig. 1 show that lexicographic and red-black GS give grid independent convergence, however, with error reduction factors that are rather bad (even for the Poisson equation, i.e. $\psi=0$ ). This is due to the fact that, in the special situation with right triangles, for the unknowns on horizontal and vertical lines we have only 3-point difference stars which is not very favourable for smoothing in $2 D$. In view of this we use a variant of Gauss-Seidel in which we use a decoupling of unknowns. This variant, which is denoted by GSD (GS with decoupling), has much better smoothing properties for our system. The following explains the GSD method. If we collect the unknowns on diagonal lines in a vector $\mathbf{x}_{\boldsymbol{d}}$ and the unknowns on horizontal or vertical lines in a vector $\mathbf{x}_{h v}$, then the system we want to solve can be written in the form

$$
\left(\begin{array}{cc}
\mathbf{D}_{1} & \mathbf{B} \\
\mathbf{C} & \mathbf{D}_{2}
\end{array}\right)\left(\begin{array}{c}
\mathbf{x}_{d} \\
\mathbf{x}_{h v}
\end{array}\right)=\left(\begin{array}{c}
\mathbf{b}_{d} \\
\mathbf{b}_{h v}
\end{array}\right) \text { with } \mathbf{D}_{1}, \mathbf{D}_{2} \text { diagonal matrices } .
$$

This system is equivalent with

$$
\left(\begin{array}{cc}
\mathrm{D}_{1}-\mathbf{B ~ D}_{2}^{-1} \mathrm{C} & \emptyset \\
\mathbf{C} & \mathbf{D}_{2}
\end{array}\right)\left(\begin{array}{c}
\mathbf{x}_{d} \\
\mathbf{x}_{h v}
\end{array}\right)=\left(\begin{array}{c}
\mathbf{b}_{d}-\mathbf{B ~ D}_{2}^{-1} \mathbf{b}_{h v} \\
\mathbf{b}_{h v}
\end{array}\right) .
$$

The 5-point stencil of the matrix $\mathbf{K}:=\mathbf{D}_{1}-\mathbf{B D}_{2}^{-1} \mathbf{C}$ and the 4-point stencil of $\mathbf{L}:=\mathbf{B D}_{2}^{-1}$ can be given a-priori. In $G S D$ we first apply a number (say $\sigma$ ) of lexicographic GS iterations 
to the system $\mathbf{K} \mathbf{x}_{d}=\mathbf{b}_{d}-\mathbf{L} \mathbf{b}_{h v}$, which results in $\mathbf{x}_{d}^{(\sigma)}$, and then we calculate an approximation of $\mathbf{x}_{h v}$ by replacing $\mathbf{x}_{d}$ by $\mathbf{x}_{d}^{(\sigma)}$. So one iteration of GSD for approximating $\left(\mathbf{x}_{d}, \mathbf{x}_{h v}\right)$ consists of the computation of $\left(\mathbf{x}_{d}^{(\sigma)}, \mathbf{D}_{2}^{-1}\left(\mathbf{b}_{h v}-\mathbf{C} \mathbf{x}_{d}^{(\sigma)}\right)\right)$.

If $\sigma=1$ then $G S D$ is just a variant of collective Gauss-Seidel. In our experiments $\sigma=2$ turned out to be a good choice (but also $\sigma=1$ is acceptable). If $\sigma=2$ then the cost of one GSD iteration is comparable with the cost of two usual Gauss-Seidel iterations.

4.5. MG algorithm. Using the bilinear forms $a_{k}(\cdot, \cdot)$, prolongations $P_{k}$ and $G S D$ smoother from above we now specify one iteration of the multigrid algorithm on level $k\left(1 \leq k \leq k_{\max }\right)$ for approximating $u_{k}^{*} \in S_{k, 0}$ which satisfies $a_{k}\left(u_{k}^{*}, \varphi\right)=l_{k}(\varphi)$ for all $\varphi \in S_{k, 0}\left(l_{k}\right.$ a given functional on $S_{k, 0}$ ).

Algorithm (level $k$ ).

Step 1. (Pre-smoothing). Apply $\nu_{1}$ iterations of GSD, resulting in $u_{k}^{\left(\nu_{1}\right)}$.

Step 2. (Coarse grid correction). Let $u_{k-1}^{*} \in S_{k-1,0}$ be such that

$$
a_{k-1}\left(u_{k-1}^{*}, \varphi\right)=l_{k}\left(P_{k} \varphi\right)-a_{k}\left(u_{k}^{\left(\nu_{1}\right)}, P_{k} \varphi\right) \text { for all } \phi \in S_{k-1,0} .
$$

If $k=1$ then $\tilde{u}_{k-1}:=u_{k-1}^{*}$. If $k>1$ then compute an approximation $\tilde{u}_{k-1}$ of $u_{k-1}^{*}$ by using $\mu=1$ or $\mu=2$ iterations of the Algorithm on level $k-1$, with starting vector 0 , applied to the problem (4.3).

Now put $u_{k}^{(\text {new })}:=u_{k}^{\left(\nu_{1}\right)}+P_{k} \tilde{u}_{k-1}$.

Step 3. (Post-smoothing). Apply $\nu_{2}$ iterations of GSD.

Remark 4.4. There is no convergence proof for the algorithm above. The convergence analysis of $[2],[3,4]$ cannot easily be modified for the situation here because the bilinear form $a_{k}(\cdot, \cdot)$ is nonsymmetric.

\section{Numerical results}

In this section we apply the algorithm of $\S 4$ to some model problems. In all experiments we use a triangulation as in Fig. 1 and a coarsest grid with $h=\frac{1}{4}$. On the finest grid we have $k_{\max }$ and $h_{\min }$ related by $h_{\min }=2^{-\left(2+k_{\max }\right)}$. We always take one pre- and one post-smoothing $\left(\nu_{1}=\nu_{2}=1\right)$. The model problems we consider are taken from the papers of Brezzi, Marini, Pietra $[5,6]$. First we consider a problem with (strong) convection in the whole domain (Experiments $1 \mathrm{~A}, 1 \mathrm{~B}$ ) and then we consider a problem with (strong) convection in part of the domain (Experiment 2). In all our experiments we measure the performance of a method by way of the average reduction factor (arf) which results by taking an arbitrary starting vector (which is the same in all experiments) and then computing the average of the norm reduction of the defect in the first 15 iterations. 
Experiment 1A. We take $f=0, \Gamma_{0}=\partial \Omega$ with $g(x, y)=1$ if $x=0$ or $y=0$ and $g(x, y)=0$ if $x=1$ or $y=1$. We take $\psi(x, y)=l(-2 x-y)$ with $l=10^{3}$.

We compare four different methods applied to (2.8) with $k_{\max } \in\{1,2,3,4,5\}$ :

1: Algorithm of $\S 4$ with $\mu=0$ (so only 2 GSD iterations on the finest grid)

2: Algorithm of $\S 4$ with $\mu=2$ (so $M G W$-cycle)

3: Algorithm of $\S 4$ with $\mu=0$ and GSD replaced by the usual lexicographic GS

4: Algorithm of $\S 4$ with $\mu=2$ and GSD replaced by the usual lexicographic GS.

The results are shown in Fig. 2.

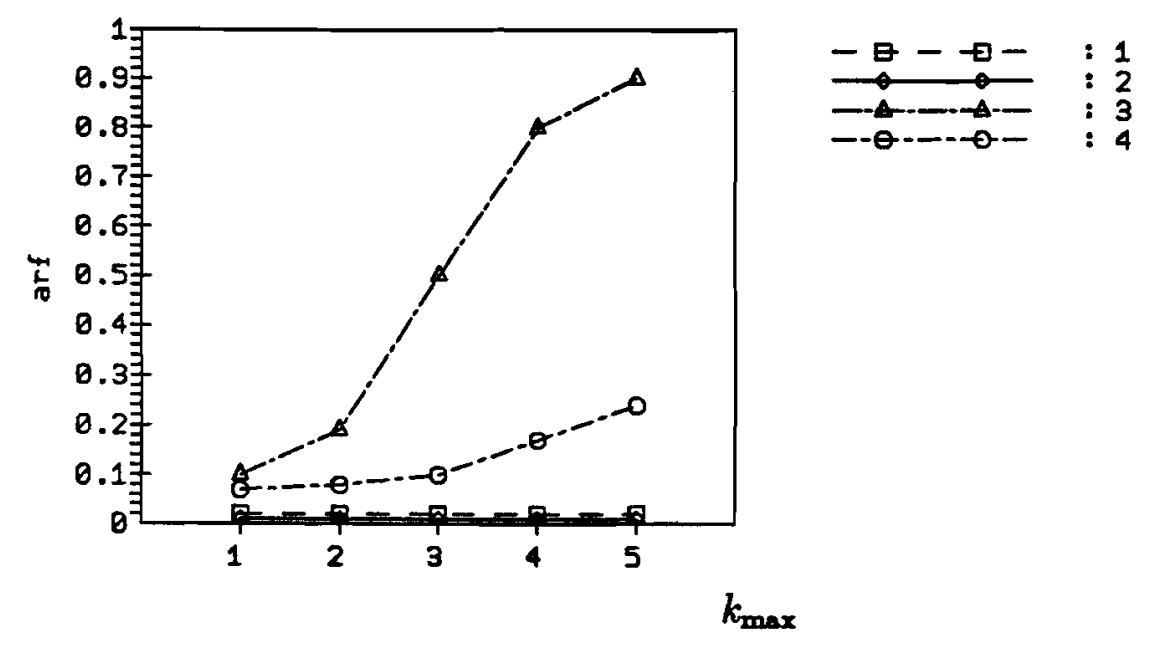

Figure 2.

In this problem with very strong convection and thus a strong upwinding effect in the discretization we expect a suitable GS iteration (without $M G$ ) to give good results. In this respect $G S D$ is preferable to lexicographic GS. We also see that the good performance of GSD is not spoiled by going to (very) coarse grids and that lexicographic GS is significantly improved by going to coarser grids.

Experiment 1B. We consider the problem as in $1 \mathrm{~A}$ but now with $l=40$ (less convection). The solution of this problem on a $16 \times 16$ grid is shown in Fig. 3. We compare the methods 1 and 2 as described in Experiment 1A. The average reduction factors are given in Table 1. 


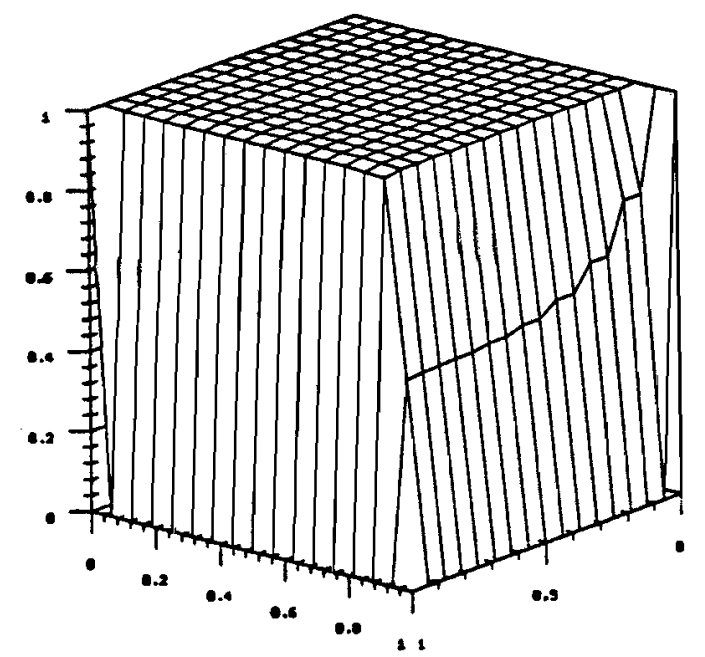

Figure 3.

\begin{tabular}{|c|c|c|c|c|c|}
\hline$k_{\max }$ & 1 & 2 & 3 & 4 & 5 \\
\hline method 1 & exact & $10^{-8}$ & $510^{-4}$ & 0.17 & 0.90 \\
\hline method 2 & exact & $10^{-8}$ & $10^{-4}$ & 0.020 & 0.058 \\
\hline
\end{tabular}

Table 1.

As expected, for $k_{\max }$ large the GSD deteriorates due to the diffusion. Using coarser grids then gives a significant improvement.

Experiment 2. From the multigrid point of view this experiment is more interesting than Experiments $1 \mathrm{~A}, \mathrm{~B}$ because here we consider a problem in which convection dominates in part of the domain and diffusion dominates in another part of the domain.

Again we take $f=0$. On part of the boundary we have Neumann boundary conditions: $\Gamma_{1}=\{(x, y) \mid((x=1)$ and $(y<0.75))$ or $((y=1)$ and $(x<0.75))\}$. On $\Gamma_{0}$ we have $g(x, y)=0$ if $x=0$ or $y=0$ and $g(x, y)=1$ otherwise. The convection is determined by the function $\psi=l \psi_{0}$ with

$$
\psi_{0}(\rho)=\left\{\begin{array}{ll}
0 & \text { if } 0 \leq \rho \leq 0.8 \\
\rho-0.8 & \text { if } 0.8 \leq \rho \leq 0.9 \\
0.1 & \text { if } 0.9 \leq \rho \leq 1
\end{array} \quad \text { with } \rho:=\left((x-1)^{2}+(y-1)^{2}\right)^{\frac{1}{2}}\right.
$$

So $\|\nabla \psi(\rho)\|_{2}=0$ if $0<\rho<0.8$ or $0.9<\rho<1$ and $\|\nabla \psi(\rho)\|_{2}=1$ if $0.8<\rho<0.9$. For $l=10^{3}$ the solution of $(2.8)$ on a $16 \times 16$ grid is shown in Fig. 4. We consider the algorithm of $\S 4$ with different values of $\mu(\mu=0,1,2)$ for problems with varying $k_{\max }\left(k_{\max }=1,2,3,4,5\right)$. For different values of $\mu$ the results for $l=10$ and $l=10^{3}$ are given in Fig. 5 and Fig. 6 respectively. 


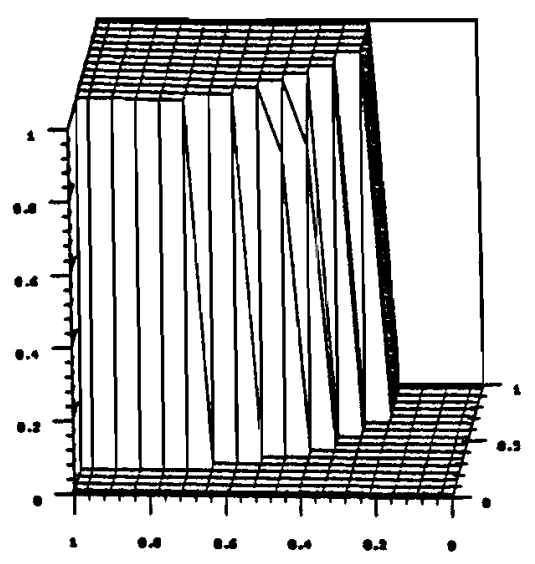

Figure 4.
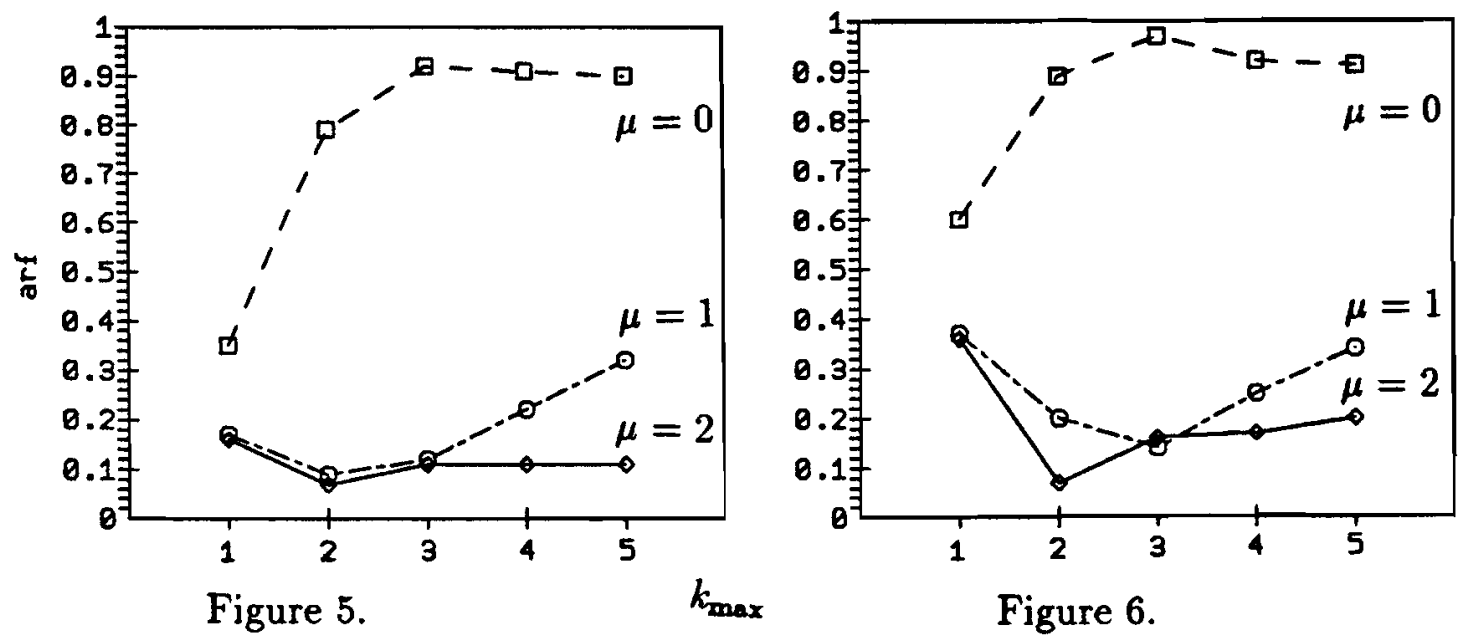

Figure 6.

In Figure 7 we show the results for $k_{\max }=4$ and with varying $\mu$ and $l$.

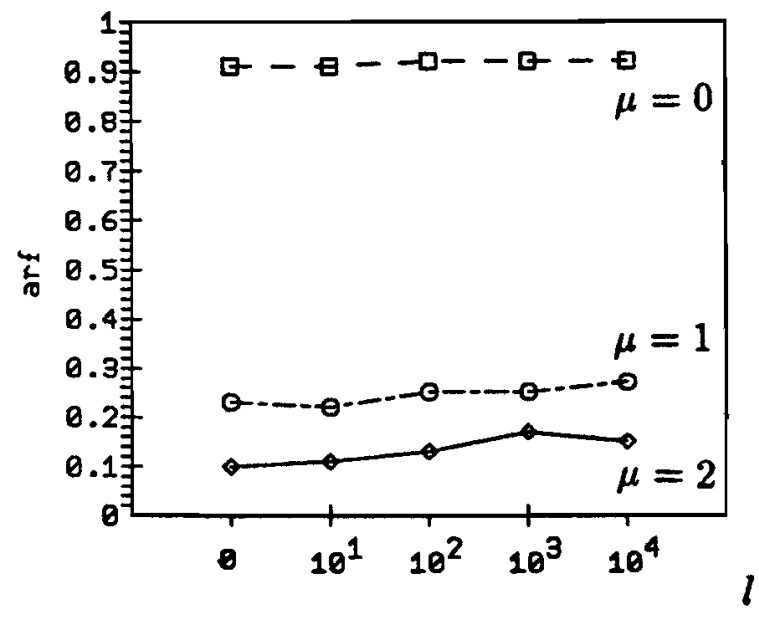

Figure 7.

These results show the typical behaviour one expects from a reasonable multigrid method: "small" (< 0.2 ) error reduction factors even for (very) fine meshes. Also our method seems to be rather robust with respect to the degree of convection present in the problem. Moreover in the multigrid method very coarse grids can be used. 


\section{References}

1. Arnold, D.N. and Brezzi, F., Mixed and nonconforming finite element methods, postprocessing and error estimates, $M^{2} A N 19$ (1985), 7-32.

2. Braess, D. and Verfürth, R., Multi-grid methods for non-conforming finite element methods, SIAM J. Numer. Anal. 27 (1990), 979-986.

3. Brenner, S.C., Multigrid Methods for Nonconforming Finite Elements, Dissertation, The University of Michigan (1988).

4. Brenner, S.C., An optimal-order multigrid method for $P 1$ nonconforming finite elements, Math. Comp. 52 (1989), 1-15.

5. Brezzi, F., Marini, L.D. and Pietra, P., Two-dimensional exponential fitting and application to drift-diffusion models, SIAM J. Numer. Anal. 26 (1989), 1342-1355.

6. Brezzi, F., Marini, L.D. and Pietra, P., Numerical simulation of semiconductor devices, Comp. Meths. Appl. Mech. and Engr. 75 (1989), 493-513.

7. Hackbusch, W., Multi-grid methods and applications, Springer, Berlin-Heidelberg (1985).

8. Marini, L.D. and Pietra, P., New mixed finite element schemes for current continuity equations, Publ. N. 695 Ist. di An. Num. Pavia (1989).

\section{A. Reusken}

Technical University Eindhoven

Department of Mathematics and Computer Science

P.O. Box 513

$5600 \mathrm{MB}$ Eindhoven

The Netherlands 
PREVIOUS PUBLICATIONS IN THIS SERIES:

\begin{tabular}{|c|c|c|c|}
\hline Number & Author(s) & Title & Month \\
\hline $90-06$ & $\begin{array}{l}\text { Y. Shindo } \\
\text { K. Horiguchi } \\
\text { A.A.F. van de Ven }\end{array}$ & $\begin{array}{l}\text { Bending of a magnetically saturated plate } \\
\text { with a crack in a uniform magnetic field }\end{array}$ & April '90 \\
\hline $90-07$ & $\begin{array}{l}\text { M. Kuipers } \\
\text { A.A.F. van de Ven }\end{array}$ & $\begin{array}{l}\text { Unilateral contact of a springboard and a } \\
\text { fulcrum }\end{array}$ & July '90 \\
\hline $90-08$ & $\begin{array}{l}\text { P.H. van Lieshout } \\
\text { A.A.F. van de Ven }\end{array}$ & $\begin{array}{l}\text { A variational approach to the magneto- } \\
\text { elastic buckling problem of an arbitrary } \\
\text { number of superconducting beams }\end{array}$ & July '90 \\
\hline $90-09$ & A. Reusken & $\begin{array}{l}\text { A multigrid method for mixed finite ele- } \\
\text { ment discretizations of current continuity } \\
\text { equations }\end{array}$ & August '90 \\
\hline $90-10$ & $\begin{array}{l}\text { G.A.L. van de Vorst } \\
\text { R.M.M. Mattheij } \\
\text { H.K. Kuiken }\end{array}$ & $\begin{array}{l}\text { A boundary element solution for } 2 \text { - } \\
\text { dimensional viscous sintering }\end{array}$ & October ' 90 \\
\hline $90-11$ & A.A.F. van de Ven & $\begin{array}{l}\text { A note on 'A nonequilibrium theory of } \\
\text { thermoelastic superconductors' by S-A. } \\
\text { Zhou and K. Miya }\end{array}$ & October ' 90 \\
\hline $90-12$ & $\begin{array}{l}\text { M.E. Kramer } \\
\text { R.M.M. Mattheij }\end{array}$ & $\begin{array}{l}\text { Timestepping for solving nonlinear equa- } \\
\text { tions }\end{array}$ & October '90 \\
\hline $90-13$ & A. Reusken & $\begin{array}{l}\text { Multigrid applied to mixed finite element } \\
\text { schemes for current continuity equations }\end{array}$ & November' 90 \\
\hline
\end{tabular}

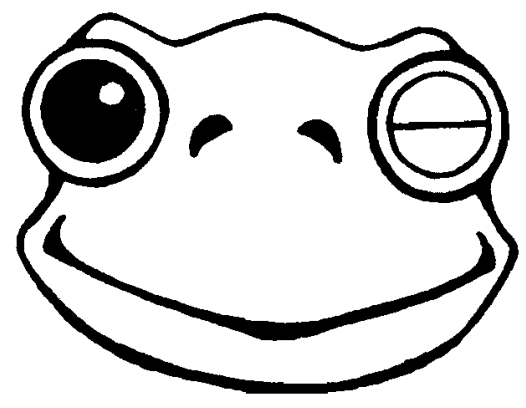

